\title{
Structural Changes in Flash Lamp Annealed Amorphous Si Layers Probed by Slow Positron Implantation Spectroscopy
}

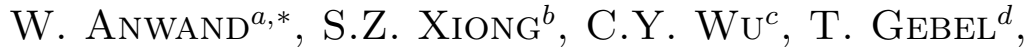 \\ Th. Schumann ${ }^{a}$, G. Brauer ${ }^{a}$ And W. Skorupa ${ }^{a}$ \\ ${ }^{a}$ Forschungszentrum Dresden-Rossendorf, PF 510119 , 01314 Dresden, Germany \\ ${ }^{b}$ Institute of Photoelectronics, Nankai University,Tianjin 300071, China \\ ${ }^{c}$ Hong Kong University of Science and Technology, Clear Water Bay, Kowloon, \\ Hong Kong, China \\ ${ }^{d}$ FHR Anlagenbau GmbH, 01458 Ottendorf-Okrilla, Germany \\ Flash lamp annealing was applied to the modification of thin amorphous \\ $\mathrm{Si}$ layers on $\mathrm{SiO}_{2}$ and glass. Slow positron implantation spectroscopy was \\ used for the characterisation of the microstructure before and after flash lamp \\ annealing. Changes in the structure down to a depth of some micrometres \\ below the surface observed with slow positron implantation spectroscopy will \\ be presented and discussed.
}

PACS numbers: 61.80.Ba, 78.70.Bj, 81.05.Ea, 81.07.Bc

\section{Introduction}

Silicon is the dominant material in the photovoltaic solar cell industry because it presents no problems regarding abundance and accessibility within the earth's crust, and generates no significant environmental problems regarding toxicity. The physical properties of Si are well investigated and understood and the essential technologies are developed, so that about $90 \%$ of the solar cells produced are currently based on Si. Due to the Shockley-Queisser limit [1], the solar conversion efficiency in single-junction photovoltaic cells is restricted to $33 \%$. However, this value is achieved neither in the laboratory nor on a technical scale. Especially, commercial solar cells show a remarkably lower efficiency depending on the quality of the Si layers, from $17 \%$ for monocrystalline layers to $7 \%$ for amorphous Si. For

${ }^{*}$ corresponding author 
this reason, great efforts are being made to improve the properties of the Si layers and to reduce the technological costs for their production. Flash lamp annealing (FLA), together with an advanced technology of the production of crystalline $\mathrm{Si}$ layers may represent a promising route towards cheaper and more effective solar cells. Starting from thin amorphous Si layers, FLA is applied to crystallise the amorphous layers and to anneal defects. Furthermore, an electrical activation of dopants might be obtained simultaneously within this ultrashort annealing.

In order to test the possible advantage of FLA outlined above, thermal treatments using FLA were applied to thin amorphous layers on glass substrates. Slow positron implantation spectroscopy (SPIS) was used before and after FLA for the characterisation of the layers and the vacancy-type defects therein. This paper summarises preliminary results of the investigations.

\section{Experimental}

Amorphous Si layers with a thickness of $100 \mathrm{~nm}$ were deposited by low pressure chemical vapour deposition (CVD) on glass substrates with a $300 \mathrm{~nm}$ thick amorphous $\mathrm{SiO}_{2}$ layer on top. The samples were annealed by FLA in order to crystallise the amorphous Si to a single-crystalline layer or to a layer composed of large single crystals. Furthermore, a very low defect concentration in the crystalline layer was aimed for.

Flash lamp annealing allows a fast heating up of solid surfaces with a single light flash between some hundred microseconds and some milliseconds. Thereby, the achievable final temperature of the layer could be more than $2000^{\circ} \mathrm{C}$ depending on the intensity of the light flash and on the optical properties of the flash lamp annealed material. Short light flashes are created in xenon gas discharge lamps emitting a spectrum in the range of the visible light, i.e. from 400 to $800 \mathrm{~nm}$ (Fig. 1). A capacitor — induction coil — arrangement delivers the required energy for the pulse lamps and allows a change of the pulse length by variation of capacitance and inductance, furthermore. In this way, the sample surfaces could be exposed with high light intensities. The reader is referred to [2] for more details about the equipment.

In case of the FLA of the $100 \mathrm{~nm}$ thick amorphous Si layers, a light flash of $20 \mathrm{~ms}$ (Fig. 2) was applied with an electrical energy of $135 \mathrm{~kJ}$ leading to an electrical power of 6.75 MW. The calculated optical energy density at the sample surface was $88 \mathrm{~J} / \mathrm{cm}^{2}$. Thus, a melting of the amorphous layer could be obtained which was visible by a yellow discoloration of the sample. Finally, the samples were slowly cooled down in order to avoid thermal stress and delaminations of the Si layer. The whole annealing procedure was carried out in an Ar atmosphere.

For the clarification of the layer properties SPIS measurements were carried out with the monoenergetic positron beam "SPONSOR" at Rossendorf, which is described in detail in [3]. About $4.5 \times 10^{5}$ events per spectrum were accumulated. The energy resolution at $E=511 \mathrm{keV}$ was $(1.09 \pm 0.01) \mathrm{keV}$. Depth depending 


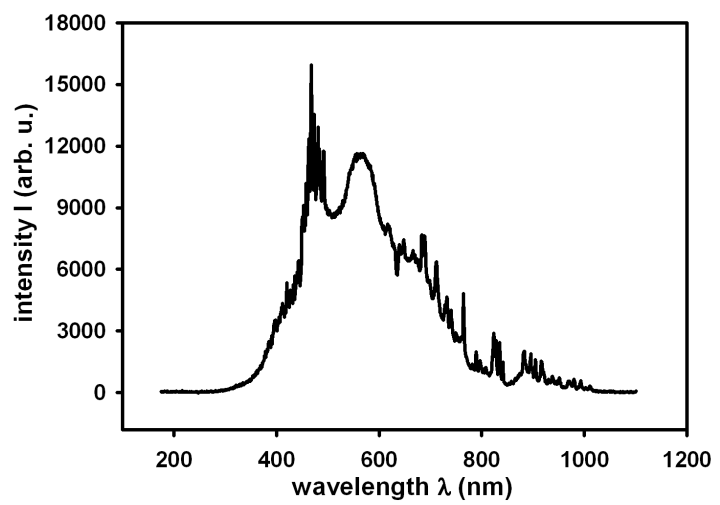

Fig. 1. Spectrum of the xenon gas discharge lamps with an electrical energy of $23 \mathrm{~kJ}$ and a pulse length of $20 \mathrm{~ms}$.

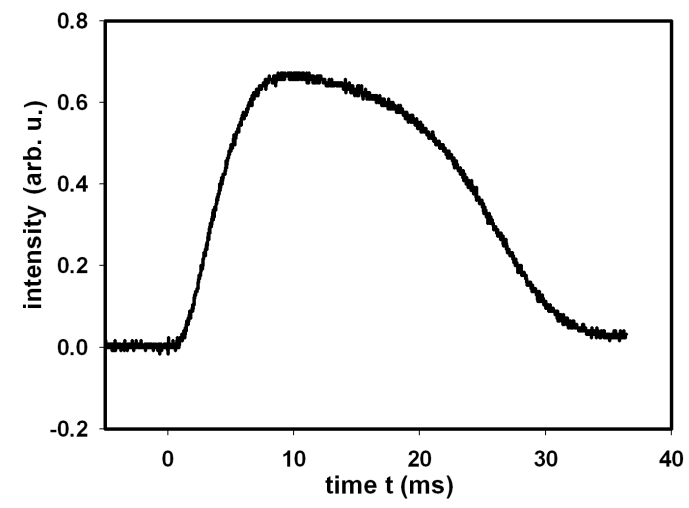

Fig. 2. Shape of a $20 \mathrm{~ms}$ light pulse.

defect profiles were calculated from the measured $S(E)$ and $W(E)$ curves using the software package VEPFIT [4].

\section{Results and discussion}

In Fig. 3, the $S$ parameter depending on the incident positron energy $E$ is shown both for the untreated and the same flash lamp annealed samples. The first small peak in the $S(E)$ curve $\left(S_{\text {amorph }}=0.4974\right)$ of the untreated sample represents the amorphous Si layer on top of the substrate. The following broader maximum $\left(S_{\mathrm{SiO}_{2}}=0.5014\right)$ is caused by the amorphous $\mathrm{SiO}_{2}$ layer, and finally the $S$ parameter meets the value of the glass substrate $S_{\text {glass }}=0.452$ at high positron energies. The minimum in the curve around $E=3 \mathrm{keV}$ is caused by the interface between the amorphous $\mathrm{Si}$ layer and the $\mathrm{SiO}_{2}$ buffer layer on top of the glass substrate. This interface does not crucially influence the properties of the investigated Si layer. For this reason, it is not discussed further. 


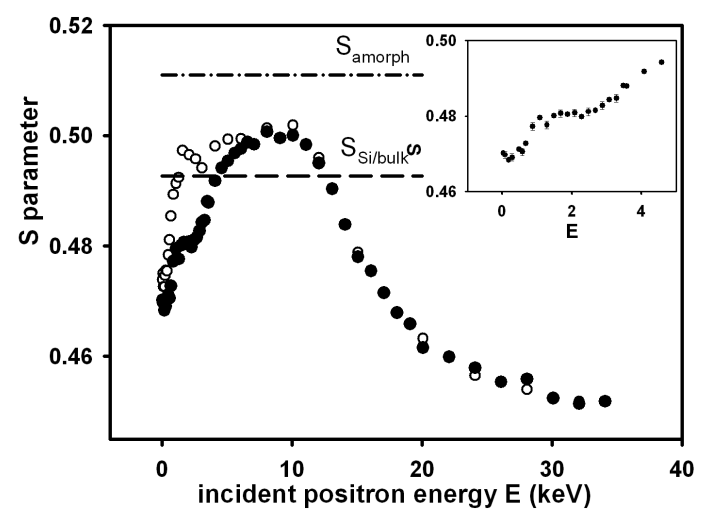

Fig. 3. $S(E)$ curves of the samples before (open circles) and after FLA (black points); the $S$ parameters of a layer amorphised by implantation (dash-dot) and $S i_{\text {bulk }}$ (longdash) are indicated together with a section of $S(0 \mathrm{keV}<E<5 \mathrm{keV})$ after FLA (top right).

The need for an amorphous $\mathrm{SiO}_{2}$ buffer layer is seen from the $S$ parameter of the glass substrate. It is considerably lower than that for amorphous material. With the procedure given in [5] it is possible to calculate the crystalline fraction of the glass substrate to $58 \%$. For this reason, an amorphous buffer layer was deposited in order to avoid partial crystalline growth of Si during CVD.

The $S$ parameter of the amorphous CVD Si layer is only $1 \%$ higher than that for Si single crystals, whereas the $S$ parameter of amorphous Si generated by ion implantation was determined to $S_{\text {amorph }} / S_{\mathrm{Si} / \text { bulk }}=1.04\left(S_{\mathrm{Si} / \text { bulk }}: \mathrm{S}\right.$ of single- crystalline bulk) [6]. This increase was mainly attributed to divacancies which were created during the implantation. This means for the CVD layer that the open volume in this amorphous configuration is only slightly increased in comparison to the crystalline structure, and larger defects are unlikely.

After FLA, a significantly decreased $S$ parameter for the Si layer $S_{\mathrm{FLA}}=$ 0.4808 was observed. This value can be seen already from the broad plateau around $E=1.8 \mathrm{keV}$ (Fig. 3, top right). The range of $S(E>8 \mathrm{keV})$ reflecting the amorphous $\mathrm{SiO}_{2}$ and the glass substrate remained unchanged, as expected. In order to get additional information about the changes in the Si layer after FLA, not only the $S$ parameter of the layer was taken into account but also its $W$ parameter was considered. Thereby, it proved helpful to use the values of $S$ and $W$ of singlecrystalline $\mathrm{Si}$ as references. The $S$ ratio was found to be $S_{\mathrm{FLA}} / S_{\mathrm{Si} / \mathrm{bulk}}=0.975$ and the corresponding $W$ ratio $W_{\mathrm{FLA}} / W_{\mathrm{Si} / \mathrm{bulk}}=1.33$. It follows from these ratios that the structure of the Si layer after FLA is completely different from a crystalline modification. The tendency of the changes in both parameters refers to an influence of oxygen on the physical properties during the annealing as shown in [7] where a decrease in $S$ and an increase in $W$ were observed for increasing oxygen influence on the structure. 
VEPFIT was used in order to calculate the boundaries of the single layers, and to verify their thicknesses assumed from the layer deposition. Another goal of the application of VEPFIT was the fit of positron diffusion lengths, especially for the Si layer after FLA. In contrast to the common procedure using $S(E)$, the $W(E)$ plots were used for the estimation of the boundaries of the single layers because of their precise reflection of the layer structure of the samples (Fig. 4). Before FLA, a thickness of the amorphous Si layer $d_{\text {amorph }}=(98 \pm 2) \mathrm{nm}$, and of the $\mathrm{SiO}_{2}$ layer $d_{\mathrm{SiO}_{2}}=(1124 \pm 33) \mathrm{nm}$ was found. After annealing, no significant change could be detected: $d_{\text {amorph }}=(83 \pm 2) \mathrm{nm}$ and $d_{\mathrm{SiO}_{2}}=(1192 \pm 19) \mathrm{nm}$. The fitted layer thickness of the amorphous $\mathrm{Si}$ is in good agreement with the expected layer thickness of the low energy CVD. However, the fit for the amorphous $\mathrm{SiO}_{2}$ resulted in a thickness of more than $1 \mu \mathrm{m}$ and differs considerably from the one initially assumed.

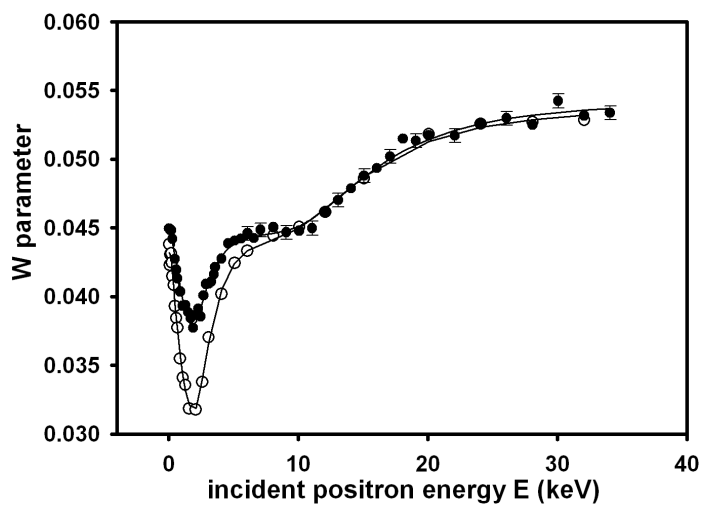

Fig. 4. $W(E)$ curves including the fitted runs (lines) before (open circles) and after FLA (black points).

The positron diffusion length in the flash lamp annealed Si layer was calculated from the $S(E)$ fit. The result of the fit confirmed the conclusion which could be drawn already from the $S(E)$ in the range $0 \mathrm{keV}<E<5 \mathrm{keV}$ (Fig. 3, top right): the broad plateau around $E=1.8 \mathrm{keV}$ indicates that all positrons annihilate in a $83 \mathrm{~nm}$ thick Si layer. This means that their diffusion length must be short. VEPFIT resulted in $L_{\mathrm{FLA}}=(5 \pm 3) \mathrm{nm}$. The positron diffusion length in Si single crystals amounts to $L_{\mathrm{Si} / \mathrm{bulk}}=250 \mathrm{~nm}$ [8]. This is a further indication that the structure of the flash lamp annealed Si layer is far away from that of crystalline Si.

\section{Conclusions}

FLA is considered to be a promising method for a thermal treatment of thin amorphous Si layers for optical applications. It was demonstrated that the energy introduced by a single light flash is high enough to affect structural changes in 
the near-surface layers without significant influence on the substrate. SPIS was used for the clarification of the structure and their changes in thin amorphous Si layers both, before and after FLA. The results have shown that the aspired crystalline structure of the top Si layer with a reduced defect concentration could not be obtained after the first attempts of FLA. The role of oxygen during the flash lamp process seems to be especially important. Further investigations are in progress in combination with other methods in order to reduce the destructive oxygen influence on the FLA of amorphous Si layers.

\section{References}

[1] W. Shockley, H.J. Queisser, J. Appl. Phys. 32, 510 (1961).

[2] http://www.fzd.de/db, search for flash lamp annealing.

[3] W. Anwand, H.-R. Kissener, G. Brauer, Acta Phys. Pol. A 88, 7 (1995).

[4] A. van Veen, H. Schut, J. deVries, R.A. Hakvoort, M.R. Ijpma, in: Positron Beams for Solids and Surfaces, Eds. P.J. Schultz, G.R. Massoumi, P.J. Simpson, AIP Conf. Proc., Vol. 218, American Institute of Physics, New York 1990, p. 171.

[5] W. Anwand, G. Brauer, M. Hasegawa, O. Dersch, F. Rauch, Acta Phys Pol. A 99, 321 (2001)

[6] R. Kögler, W. Anwand, G. Brauer, A. Peeva, W. Skorupa, P. Werner, U. Gösele, Appl. Phys. Lett. 75, 1279 (1999).

[7] G. Brauer, F. Becvar, W. Anwand, W. Skorupa, Appl. Surf. Sci. 252, 3368 (2006).

[8] R. Krause-Rehberg, H.S. Leipner, Positron Annihilation in Semiconductors Defect Studies, 1st ed., Springer-Verlag, Berlin 1999, p. 148. 